\title{
The Best Posters from the 23rd Annual Scientific Meeting of the International Society of Cardiovascular Pharmacotherapy (ISCP)
}

Kyoto, Japan

26-27 May 2018

A total of 64 general abstracts were submitted, with 14 abstracts selected by the judges as candidates for the Best Abstract Awards, which are presented here. Of these 14 abstracts, three were recipients of an award.

\section{Best Abstract Award - First prize Topic: Heart Failure (Basic), Molecular Cardiology}

\section{Cavin-2 Deficiency Attenuates Cardiac Fibrosis and Dysfunction in Pressure-overloaded Hearts}

\author{
Yusuke Higuchi, ${ }^{1}$ Takehiro Ogata, ${ }^{2}$ Masahiro Nishi ${ }^{1}$ and Satoaki Matoba ${ }^{1}$ \\ 1. Department of Cardiovascular Medicine, Kyoto Prefectural University of Medicine; 2. Department of Pathology and Cell Regulation, Kyoto \\ Prefectural University of Medicine, Kyoto, Japan
}

Keywords: Heart failure, Cavin-2 deficiency, cardiac fibrosis, systolic dysfunction, fibroblasts, myofibroblasts Citation: European Cardiology Review 2018;13(2):120. DoI: https://doi.org/10.15420/ecr.2018.13.2.P01

\section{Introduction}

Heart failure (HF) is a debilitating disease associated with high morbidity and mortality. The high mortality rate reflects inadequacy of modern therapy and calls for new mechanistic treatments. A major cause of $\mathrm{HF}$ is the adverse tissue remodelling with fibrosis. Excessive extracellular matrix (ECM) turnover is involved in poor outcome. Transdifferentiation of fibroblasts into activated myofibroblasts is a defining feature of fibrosis. Myofibroblasts express alpha-smooth muscle actin and secrete ECM proteins. Previous report showed that the cavin family gene is involved in cardiac function. However, the role of Cavin-2 in cardiac fibrosis and cardiac function remains unknown.

Methods and Results

To examine the role of Cavin-2 in cardiac function, transverse aortic constriction (TAC) was performed on wild type (WT) mice and Cavin2-/- mice. Four weeks after TAC procedure, left ventricular fractional shortening was significantly preserved in Cavin-2-/- mice. In addition, the fibrosis area was significantly attenuated in Cavin-2-/- mice. Moreover, mRNA expression of fibrosis genes was attenuated in the hearts of Cavin-2-/- mice four weeks after TAC. In in vitro study, we isolated mouse embryonic fibroblasts (MEFs) from Cavin2-/- mice and WT mice. Western blotting showed that alphasmooth muscle actin protein level was decreased in TGF-alpha-1 stimulated MEFs derived from Cavin-2-/- mice. Furthermore, mRNA expression of fibrosis genes was attenuated in MEFs derived from Cavin-2-/- mice.

\section{Conclusions}

Our observations suggest that Cavin-2 contributes to the development of cardiac fibrosis and systolic dysfunction with the differentiation of fibroblasts into myofibroblasts. Cavin-2 may be a novel therapeutic target for cardiac fibrosis. 